\title{
La relación docente-asistencial en el nuevo contexto que establece la reforma de salud
}

\section{O scar Román A, Miriam Señoret $\mathrm{S}^{\mathrm{a}}$. \\ The relationship between health care and teaching activities in the context of the Health Care Reform}

The new laws of the health reform in Chile, modified the preexistent relationship between teaching and health care activities. This relationship allows the training of health care professionals by Universities, in the clinical services of the National System of Health Services that is dependent of the Ministry of Health. The new law established new requirements for specialization and quality of care accreditation. Up to the moment, the relationship between Health Care Services and the Universities was based on harmonic and consensual agreements between both counterparts. With the new law, such relationship becomes more relative and is based on new norms. Therefore, it is of utmost importance to establish a regulated and equilibrated relationship between both institutions, to assure the best possible training of health care professionals, in terms of skills and competences, to adequately satisfy the health needs of the population (Rev Méd Chile 2007; 135: 251-56).

(Key words: Accreditation; Education, medical; Health care reform)

Recibido el 15 de junio, 2006. Aceptado el 19 de julio, 2006.

Departamento de Medicina, Campus Centro, Facultad de Medicina, Universidad de Chile y Ministerio de Salud. División de Gestión y Desarrollo de las Personas.

aMatrona

L a promulgación de las nuevas leyes sobre Estatuto Médico Funcionario diurno (№ 19.664) ${ }^{1}$, Autoridad Sanitaria (№ 19.937) ${ }^{2}$ y Garantías en Salud (Auge) (№ 19.996) ${ }^{3}$, vigentes desde los años 2000 a 2004, ha determinado diversos cambios en la relación entre el Ministerio de Salud y sus servicios con las carreras de la salud de

Correspondencia a: Dr. Oscar Román A. Huelén 154 Dpto. 2 Providencia, Santiago, Chile. E mail: oromanalemany@gmailcom universidades e institutos de educación superior. Las nuevas disposiciones, tanto legales como administrativas, pueden producir mejoras en la relación docente-asistencial (DA) como también aspectos controversiales de diversa índole que es preciso analizar.

En nuestro medio se reconoce que, en función de los criterios actuales de competencia en la docencia universitaria y técnica ${ }^{4,5}$, la preparación de los estudiantes debe ser realizada en los campos clínicos, tecnológicos y administrativos de los hospitales y consultorios de los Servicios de Salud. Por ello, es de interés analizar el nuevo 
marco legal y administrativo que las leyes señaladas imponen al sistema sanitario y su repercusión sobre la docencia del recurso humano de salud.

Históricamente, la relación entre el estado y las universidades con sus carreras de medicina se inició en el año 1833, mediante el Decreto № 1329 del 19 de marzo de ese año, que creó la Escuela de Medicina en el Instituto Nacional ${ }^{6}$. Posteriormente, las clases se trasladaron a los Hospitales San Juan de Dios y San Vicente, junto a la enseñanza práctica y los internados. Si bien desde esa época, la «ocupación» docente de los grandes hospitales del país era una realidad, ella se instituyó sólo en 1942 mediante una «omisión Nacional Docente-Asistencial», que estableció que la Jefatura de los Servicios Clínicos de los 4 principales hospitales de Santiago debía ser ejercida por los Profesores Titulares de la Universidad de Chile en el ramo. Así ocurrió con los Servicios de Medicina Interna, Cirugía General, Pediatría y Obstetricia $^{6}$. Esta disposición administrativa rindió frutos, por cuanto permitió concentrar en una sola mano la gestión docente y asistencial en los principales servicios clínicos de la capital.

Posteriormente, en 1952, con la instauración del Servicio Nacional de Salud (SNS), se integraron a su consejo directivo dos profesores de la Universidad de Chile (decano y otro profesor titular), lo que permitió la participación docente en el diseño y programación de las políticas de salud y de formación de los profesionales necesarios para cumplirlas.

Sin embargo, sólo en 1974 se estructuró legalmente la Comisión Nacional Docente-Asistencial (CONDAS), mediante el Decreto № 750 del Minsal ${ }^{7}$, perfeccionada por el Decreto Supremo 972 de $1992^{8}$, quedando establecida como entidad asesora del ministro del ramo y formada por los decanos de las Facultades de Medicina tradicionales y dos representantes del Colegio Médico. Además, cuando las materias a resolver no correspondieran a la profesión médica, se facultó la incorporación a ella del decano de la escuela profesional correspondiente. En el mismo Decreto se establecieron también comisiones D-A regionales (CORDAS) y locales (COLDAS) que, en las regiones y hospitales locales, debían representar a la entidad nacional. Ellas serían dirigidas por el Secretario Regional Ministerial y por los directores de hospital, respectivamente ${ }^{8}$.
Esta estructuración ha estado vigente hasta el presente y ha demostrado cierta efectividad para solucionar los problemas de la relación D-A a nivel general y local. Es necesario reconocer, sin embargo, que el porcentaje de constitución y acción de las CORDAS ha sido bajo, no así el de las COLDAS, que ha sido significativo (mayor de $50 \%)^{9}$.

En 2001, como acuerdo de CONDAS, refrendado por el Ministro de Salud de la época, se instituyó la Norma Administrativa № 7, que estableció regulaciones en la asignación de campos clínicos de los Servicios de Salud a las Universidades ${ }^{10}$. Sin embargo, ella ha tenido, limitado cumplimiento por parte de los Directivos de los Servicios de Salud.

Describiremos a continuación, en forma objetiva, la situación legal y administrativa preexistente y los cambios determinados por la nueva legislación.

\section{CAMBios de la RELACIÓN D-A CON la ReForma de SALUd}

Desde 1990 hasta la actual reforma, la relación docente-asistencial presentaba diversas características legales y administrativas que, con la nueva legislación vigente, han sufrido modificaciones que involucran tanto a las instituciones pactantes como a los profesionales respecto a su especialización y acreditación.

A) Actores institucionales en los Convenios D-A. La firma de convenios entre el Ministerio de Salud y las universidades estaba radicada jurídicamente sólo entre los directores de los 28 Servicios de Salud y los rectores de las universidades y circunscrita casi exclusivamente a los campos clínicos de los hospitales base. La Ley de Autoridad Sanitaria, en su Art. 16 bis 2, define para cada Servicio de Salud (SS) una red asistencial, constituida por el conjunto de establecimientos públicos administrados por él e integrada además por los establecimientos municipales de Atención Primaria de Salud y privados (clínicas, hospitales, centros médicos) situados en su jurisdicción territorial, siempre que estos últimos suscriban convenio con el SS. En el mismo artículo se agrega que cualquiera de los establecimientos autorizados con convenio, ya mencionados, sprestarán atención de salud progra- 
mada y de urgencia, además de acciones de apoyo y docencia cuando correspondiere, con los recursos físicos y humanos de que dispongan», pudiendo realizar determinadas acciones convenidas en postas, estaciones médicas u otros establecimientos autorizados».

De esa forma, los directores de los SS pueden extender su atribución de firmar convenios de gestión asistencial y por extensión docente, con otros establecimientos de la red, sean municipales 0 privados.

Además, el artículo 25 A de la misma Ley 2, estipula que los establecimientos hospitalarios que tengan mayor complejidad técnica, desarrollo de especialidades y organización administrativa y que adquieran la calidad de Establecimientos Autogestionados», podrán "celebrar convenios de cualquier naturaleza, independientemente del servicio al que pertenecen». Se denominan establecimientos autogestionados en red» aquellos hospitales base u otros de gran complejidad (56 en todo el país) que adquieran tal calidad en base a un proceso de acreditación regulado. Ellos deberán realizarlo en los próximos años, hasta el 2009, fecha en que todos serán acreditados por el solo Ministerio de la Ley².

Los establecimientos autogestionados podrán «celebrar contratos de compra de servicios de cualquier naturaleza, con personas naturales o jurídicas, para el desempeño de todo tipo de tareas o funciones, generales o específicas, aun cuando sean propias o habituales del establecimiento». Además podrán ejecutar y celebrar toda clase de contratos y actos sobre bienes muebles e inmuebles y sobre cosas corporales o incorporales que hayan sido asignadas 0 afectadas al establecimiento».

De acuerdo a esa norma, esos hospitales podrán efectuar convenios con entidades docentes, como universidades, carreras de la salud e institutos de educación superior, en cuanto se refiere a ocupar sus dependencias y las de la red correspondiente. La única salvedad es que aquellos convenios con entidades que no sean parte de su red asistencial, como son los establecimientos docentes, deberán contar con la aprobación del respectivo Director del Servicio de Salud.

B) Actores personales o recurso humano. La situación previa a la reforma permitía que los funcionarios del SS (médicos u otros profesionales), pudieran tener cargos mixtos docente-asistenciales en los establecimientos afectos a los convenios D-A, siempre que la suma de ambas jornadas no excediera 44 horas semanales. El artículo 82 de la Ley de Autoridad Sanitaria ${ }^{2}$ establece que los profesionales contratados no podrán tener menos de 22 horas semanales y que un mismo funcionario no podrá serlo por más de 44 horas en total, para lo cual, se considerarán todos los nombramientos que posea en cualquier órgano de la Administración del Estado. Sin embargo, la Ley 19.664, que modificó el estatuto médico funcionario ${ }^{1}$, permite una extensión horaria de 11 horas semanales para los médicos funcionarios que desempeñen además docencia en horas contratadas por universidades o institutos de educación superior que hayan celebrado convenios D-A vigentes.

C) Acreditación de los SS. La «acreditación» de las actividades de los SS, definida como eel procedimiento de evaluación de los recursos institucionales tendiente a garantizar la calidad de la atención a través de estándares previamente aceptados», no estaba normada en la época anterior a la reforma. En la actualidad se está trabajando en este proceso ${ }^{10}$. Las universidades han tenido sólo participación en la definición de normas de calidad requeridas por Minsal y algunas comisiones locales (COLDAS) en hospitales A y B.

La actual Reforma, en el Art. $1^{\circ}$ de la Ley de Autoridad Sanitaria, establece que sal Ministerio de Salud le corresponde velar por el cumplimiento de las normas en materia de salud y que la labor de inspección y verificación del cumplimiento de dichas normas podrá ser encomendada a terceros idóneos, debidamente certificados conforme a reglamento». Tales entidades externas deberán cumplir los siguientes requisitos: experiencia calificada de a lo menos tres años en la materia, personal idóneo e infraestructura suficiente. En caso de que estas actividades puedan ser desarrolladas por universidades, łas bases de licitación deberán considerar esta condición con un mayor factor de ponderación».

D) Asignación y uso de campos clínicos de los SS. La utilización de los campos clínicos de los Servicios de Salud por las universidades e institutos de educación superior para desarrollar docen- 
cia y establecer las necesarias competencias de las distintas profesiones ${ }^{4,5}$ existe desde el siglo XIX, habiendo sido institucionalizada en 1942 con la creación de la primera Comisión Nacional D-A ${ }^{6}$. Sin embargo, hasta 2001 no existía una normativa explícita al respecto. En ese año, con la participación de la Asociación de Facultades de Medicina (ASOFAMECH), el Ministerio de Salud aprobó la «Normativa administrativa № 711, que estableció modalidades y conceptos generales que regulaban la asignación de campos clínicos de los SS a las universidades y centros formadores con el objeto que ellos realizaran la práctica docente en función del tipo de médico que el país necesitaba ${ }^{12}$. Sin embargo, algunos conceptos de dicha normativa han resultado conflictivos, como la denominada no cohabitación»o exclusividad de la utilización de un campo clínico por una determinada universidad o carrera de la salud y también la compensación económica que exigen algunos SS. Esta compensación financiera estaría justificada en función de los gastos que pudiesen ocasionar las actividades docentes (insumos, uso de equipos y laboratorios, etc.). En este aspecto, las universidades hacen presente que ellas colaboran en la labor asistencial no sólo con académicos, docentes y becados, sino también con equipos, laboratorios y estructuras inmuebles diversas.

Esta controversia está aún vigente, por lo que actualmente el Ministerio de Salud, en conjunto con las universidades, estudia una nueva normativa que permita una flexibilización de las exigencias respecto a la utilización de los campos clínicos por aquellas, sean públicas o privadas.

E) Especialización. En la situación previa a la reforma no existían normativas ni ley que regulara la especialización médica o de las otras carreras de la salud. Cada profesional, con su título legalmente reconocido, podía ejercer cualquier especialidad que lo motivara, sin presentar certificación al respecto ${ }^{13}$. Sin embargo, desde la década de 195059, el Servicio Nacional de Salud estableció becas para las especialidades madres o clásicas (Medicina Interna, Cirugía general, Pediatría y Obstetricia), denominadas Becas Primarias, las que, después de 3 años de formación, concedían la certificación de especialista. Con respecto a las otras especialidades, la certificación fue entregada, a partir de 1984, a la Comisión Nacional de Certificación de Especia- lidades Médicas (CONACEM) ${ }^{14}$, institución privada de derecho público, en la que participaban el Ministerio de Salud, las diversas sociedades científicas, las universidades y el colegio médico. Ella ha permitido regular la certificación de las diversas especialidades médicas, sean básicas, primarias 0 subespecialidades.

Otro paso importante en este proceso ha sido la concesión de título de especialista por las Escuelas de Graduados de las universidades tradicionales, mediante programas validados» de formación en diversas especialidades, con una duración de 2 a 3 años ${ }^{15}$.

Actualmente, la nueva Ley de Autoridad Sanitaria, en el párrafo 13 de su Art. $1^{\circ}$, establece que Minsal «deberá establecer un sistema de certificación de especialidades y subespecialidades de los prestadores individuales de salud legalmente habilitados, esto es, de las personas naturales que otorgan prestaciones de salud.» Agrega que «en conjunto con educación, se determinarán las entidades públicas y privadas, nacionales o internacionales, que certificarán las especialidades 0 subespecialidades, como así mismo las condiciones generales que ellas deberán cumplir con el objetivo de recibir la autorización para ello» El reglamento de la Ley 16 sestablecerá las condiciones de los certificadores como de las especialidades que serán objeto de este proceso. El registro lo deberá mantener la Superintendencia de Salud.»

En el párrafo siguiente se establece que das universidades reconocidas oficialmente en Chile, serán entidades certificadoras respecto de los alumnos que hayan cumplido un programa de formación y entrenamiento ofrecido por ellas mismas, si los programas de especialización correspondientes se encuentran acreditados en conformidad con la normativa vigente». Ello confiere poder legal a las universidades en la acreditación de especialidades y no sólo de títulos profesionales $^{15}$.

Otra normativa legal que influye en el proceso de especialización es la instituida por la Ley 19.664¹, que modificó el Estatuto Médico funcionario (Ley 15.076). Ella establece que la primera etapa de la carrera funcionaria, denominada de «estinación y Formación», que significa un contrato por 9 años en atención primaria, permitirá al cabo de un mínimo de 3 años, acceder a programas de perfeccionamiento o especialización que 
ofrezcan los Servicios de Salud o Minsal. Los Programas de Especialización «serán los necesarios para satisfacer las necesidades de los respectivos SS». Para su cumplimiento, el Art. 3 de la ley establece que los servicios podrán celebrar convenios entre sí o con otros centros formadores. En la práctica, los convenios se están realizando con las universidades regionales respectivas.

F) Calidad de la atención y su acreditación. Antes de la reforma no existían normas legales ni reglamentarias orientadas a velar por la calidad de la prestación asistencial. Sin embargo, han estado vigentes normas éticas, establecidas por los colegios profesionales y administrativas en las instituciones públicas (SS y otros) y privadas (clínicas, hospitales docentes, etc.).

El Decreto Ley 2763, refrendado por la Ley 19.937, en su Art. 4, numeral 142, determina que, mediante resolución, se establecerán protocolos de atención en salud». Estos serán instrucciones sobre manejo operativo de determinados problemas de salud», en clara referencia a los incluidos en la Ley de Garantías o Auge $^{3}$. Tales protocolos serán de carácter referencial y sólo obligatorios para el sector público y privado en caso que exista una causa sanitaria que lo amerite, lo que deberá constar en una resolución de Minsal. En la confección de estos protocolos, en particular los referidos al Auge, se ha llamado a colaborar a algunas universidades.

En el Reglamento de la Ley de Autoridad Sanitaria, en su Art $4^{\circ} 15$, se establece que el Ministerio de Salud «dictará las normas a través de las cuales establecerá criterios y parámetros de calidad de los programas que requiera. Además, evaluará la capacidad de los centros formadores para ejecutar tales programas, considerando, entre otros aspectos, la malla curricular y los campos clínicos de que dispongan.

Esta disposición reglamentaria confiere poder a Minsal para influir en la acreditación de programas, currículos y otras características de las universidades y centros formadores que deseen establecer convenios D-A con sus SS.

\section{DisCUSIÓN Y COMENTARIO}

La relación entre universidades y centros formadores del personal de la salud con el ministerio del ramo, principal empleador de ese recurso humano, ha constituido un problema significativo en las últimas dos décadas, debido básicamente a que ambos sectores, educación y salud, tienen prerrogativas, leyes e intereses distintos, que no han podido ser armonizados integralmente. A pesar de ello, ambos actores han debido necesariamente establecer contactos serios y profundos para enfrentar la preparación del recurso humano en salud. Ello se ha logrado en base a «convenios») a veces de tipo administrativo y otros de tipo legal, de acuerdo a las prerrogativas que ambas instancias poseen. Gracias a esta interrelación, las universidades y centros formadores técnicos y profesionales han formado un personal de salud de perfil adecuado a las necesidades sanitarias del país, tanto en conocimientos como en competencia de manejo y tratamiento, utilizando los campos clínicos de los $\mathrm{SS} 4,5,10-12$.

Sin embargo, persisten carencias en el proceso de formación del recurso humano, como la ausencia de una adecuada planificación a corto y mediano plazo ${ }^{17}$, y la adecuada complementación en la asignación y uso de los campos clínicos. Actualmente se trabaja en perfeccionar y actualizar una normativa administrativa que regule dicha relación.

Por otro lado, el panorama educacional ha cambiado por la emergencia de numerosas universidades privadas con carreras de la salud, lo que ha determinada actualmente una necesidad creciente de campos clínicos asistenciales para ellas.

Además, con la reforma se han establecido cambios legales y reglamentarios que regularán la especialización de los profesionales, la acreditación de los campos clínicos de los SS y la certificación de preparación y calidad de los prestadores de salud $12,14,15,18$.

Otro elemento a considerar es el sector privado de salud, que se ha expandido en forma importante, usufructuando de la formación integral de sus profesionales proporcionada por las universidades públicas o con subsidio estatal. Por tanto, en función de la asistencia sanitaria nacional, es conveniente precisar el recurso humano que ambos sectores, público y privado, requieren tanto en número como en condiciones de especialización y certificación de calidad ${ }^{17}$.

Debido a la influencia que los cambios legales y administrativos señalados van a tener sobre la 
interrelación docente asistencial, es fundamental que Minsal y centros formadores en general, sean capaces de asumir una responsabilidad compartida en el logro de un mejor entendimiento y solución de los problemas y controversias que hemos señalado. Urge en consecuencia, establecer bases claras y normas adecuadas que regulen la forma-

\section{REFERENCIAS}

1. Diario Oficial. Ley 19.664. Establece Normas especiales para profesionales funcionarios que indica en los Servios de Salud y modifica la Ley 15.076. XI-2000.

2. Diario Oficial. Ley 19937. Modifica el D.L. № 2.763 de 1979, con la finalidad de establecer una nueva concepción de la autoridad sanitaria, distintas modalidades de gestión y fortalecer la participación ciudadana. II, 2004.

3. Diario Oficial. Ley 19.669. Establece un régimen de garantías en salud. IX, 2004.

4. Declaración Conjunta de los Ministros Europeos de Educación. Declaración de Bolonia. 19-junio, 1992. www.UMCE.cl

5. SAntos L, GonzálEZ C. El diseño curricular por competencia en la educación médica. Escuela Nacional de Salud Pública. Ciudad de la Habana www.bvs.sld.cu/ revista/ENS 04403.htn. Vol 17/04-2003.

6. Escuela de Medicina. Historia de la Medicina. Reseña histórica de la formación médica en Chile. www.escuela.med.puc.cl. 2004.

7. Decreto Oficial № 750. Ministerio de Salud. Crea la Comisión Nacional Docente-Asistencial (CONDAS). I,1974.

8. Decreto Supremo 972. Ministerio de Salud. Reestructura la Comisión Nacional Docente-Asistencial CONDAS. 1992.

9. Román O, Yevenes S, Guerra M. Estudio acerca de la situación actual de la relación docente-asistencial. Monografía, Minsal. 2000.

10. Ministerio de Salud. Reforma del Sistema de Salud. Discurso estratégico. Actas del Seminario ción de un recurso profesional y técnico de salud óptimo en número y calidad para cumplir los objetivos de la reforma de salud. Para ello es de toda conveniencia establecer una relación D-A armónica que permita obtener el mejor perfil posible de los profesionales de la salud en función de una atención óptima de la población del país.

Franco-Chileno sobre reforma en los servicios hospitalarios y recursos humanos. Editorial IKKON. Rennes. Francia, págs. 19-27.

11. Norma General Administrativa. Ministerio de Salud. Regulaciones en materia de asignación y uso de los campos clínicos en los Servicios de Salud 2001.

12. Carabantes J. Sistema público de salud y tipo de médicos que éste necesita. Rev Méd Chile 1997; 125: 24-36.

13. Aguire Moreno E. Condición jurídica de la formación, reconocimiento y control del ejercicio de las profesiones médicas y paramédicas. Informe Asesoría Jurídica. Ministerio de Salud, 1999.

14. Comisión Nacional de Certificación de Especialidades Médicas (CONACEM). Evaluación de 10 años de experiencia de la Corporación Autónoma de Certificación de Especialidades Médicas. Ed. Alerce Santiago Chile. 1994.

15. Rosselot E, Mateluna E, Estevez A, Radrigán ME. Acreditación de títulos profesionales e instituciones formadoras: el caso de Chile. Rev Méd Chile 1998; 126: 715-24.

16. Decreto Supremo 128. Ministerio de Salud. Aprueba reglamento sobre sistema de acreditación a que se refieren los artículos 16 y siguientes de la Ley 19.664. VIII, 2004.

17. HaLL T. Informe taller «Modelos de simulación y planificación en Recursos Humanos». Monografía. Ministerio de Salud, Marzo de 2004.

18. Torres C, Solmano G. Formación de médicos especialistas en el contexto de la Reforma de Salud. Monografía Escuela de Salud Pública, 2005. 